\title{
Exponential and polynomial dichotomies of operator semigroups on Banach spaces
}

\author{
by \\ Roland Schnaubelt (Halle)
}

\begin{abstract}
Let $A$ generate a $C_{0}$-semigroup $T(\cdot)$ on a Banach space $X$ such that the resolvent $R(i \tau, A)$ exists and is uniformly bounded for $\tau \in \mathbb{R}$. We show that there exists a closed, possibly unbounded projection $P$ on $X$ commuting with $T(t)$. Moreover, $T(t) x$ decays exponentially as $t \rightarrow \infty$ for $x$ in the range of $P$ and $T(t) x$ exists and decays exponentially as $t \rightarrow-\infty$ for $x$ in the kernel of $P$. The domain of $P$ depends on the Fourier type of $X$. If $R(i \tau, A)$ is only polynomially bounded, one obtains a similar result with polynomial decay. As an application we study a partial functional differential equation.
\end{abstract}

1. Introduction and preliminaries. Exponential stability and dichotomy are among the most basic and most important asymptotic properties of a strongly continuous operator semigroup $T(\cdot)$ on a Banach space $X$. One strives to characterize these notions in terms of the generator $A$ of $T(\cdot)$ which is the given object in most applications. There is a well developed theory for this problem which nevertheless does not answer several important questions. In this paper we want to address one of these open issues, treating also polynomial dichotomies within the same approach.

To provide the background for our main theorems, let us describe the relevant known results in this area. Our notation is explained at the end of this section. We first recall the well known resolvent estimates which are necessary for exponential stability and dichotomy:

$$
T(\cdot) \text { is exponentially stable }
$$

$$
\Rightarrow s(A)<0 \text { and }\|R(\lambda, A)\| \leq c, \operatorname{Re} \lambda \geq 0,
$$

$T(\cdot)$ is exponentially dichotomous

$$
\Rightarrow i \mathbb{R} \subset \varrho(A) \text { and }\|R(i \tau, A)\| \leq c, \tau \in \mathbb{R} .
$$

2000 Mathematics Subject Classification: Primary 47D06.

Key words and phrases: dichotomy, splitting projection, Fourier type, strongly continuous semigroup, Gearhart's theorem, Weis-Wrobel theorem, resolvent estimates, fractional powers, delay equation. 
If $t \mapsto T(t)$ is continuous in the operator norm for some $t>0$, then the pure spectral criteria $s(A)<0$, resp. $i \mathbb{R} \subset \varrho(A)$, already imply the exponential stability, resp. dichotomy, of $T(\cdot)$ (see e.g. [8, Cor. IV.3.11, Thm. V.1.17]). In this way exponential stability and dichotomy of, e.g., analytic semigroups can be characterized very conveniently. Unfortunately, for general semigroups these criteria fail even on a Hilbert space $X$ (see [4, Ex. 5.8], [5, §2.1.5], [8, §IV.3.a], [19, Ex. 1.2.4]). (Some of these examples arise from wave equations.) On the other hand, on a Hilbert space $X$ Gearhart's spectral mapping theorem establishes the converse implications in (1.1) and (1.2); i.e.,

$T(\cdot)$ is exponentially stable

$$
\Leftrightarrow s(A)<0 \text { and }\|R(\lambda, A)\| \leq c, \operatorname{Re} \lambda \geq 0,
$$

(1.4) $T(\cdot)$ is exponentially dichotomous

$$
\Leftrightarrow i \mathbb{R} \subset \varrho(A) \text { and }\|R(i \tau, A)\| \leq c, \tau \in \mathbb{R},
$$

if $X$ is a Hilbert space (see e.g. [5, $\S 2.1],[19$, Thm. 2.2.4], and [19, p. 70] for further references). However, on non-Hilbertian $X$ there are semigroups violating the implications " $\Leftarrow$ " in (1.3) and (1.4) (see [5, §2.1.5], [8, §IV.3.a], [19], $[25, \S 4])$.

Going back to the general case of a $C_{0}$-semigroup on a Banach space $X$ without additional regularity properties, one can look for stronger assumptions on the resolvent which imply the exponential stability or dichotomy of $T(\cdot)$. Such conditions were found in [5, §2.2], [11]-[14], [18, Thm. A-III-7.10], [24]. However, they are quite sophisticated and (it seems) difficult to check in applications. Alternatively, one can ask whether the uniform boundedness of $R(\lambda, A)$ for $\lambda \in i \mathbb{R}$ or $\operatorname{Re} \lambda \geq 0$ implies interesting asymptotic properties of the semigroup which are related to stability or dichotomy. In the case of exponential stability this question was settled in a line of research culminating in the paper [25] by Weis and Wrobel (see also [19], [20], [23], [24], and the references therein). Theorem 3.2 and Remark 3.3 of $[25]$ (or [19, Thm. 4.2.4]) show that

$$
\|R(\lambda, A)\| \leq c, \operatorname{Re} \lambda \geq 0 \Rightarrow\|T(t) x\| \leq M e^{-\varepsilon t}\left\|(w-A)^{\beta} x\right\|, t \geq 0,
$$

for $\beta=1 / p-1 / p^{\prime}, x \in \mathcal{D}\left((w-A)^{\beta}\right)$, and some constants $M, \varepsilon>0$. Here $w$ is a fixed real number larger than the growth bound of $T(\cdot), p^{\prime}=p /(p-1)$, and $p \in[1,2]$ is the Fourier type of $X$, i.e., the Fourier transform $\mathcal{F}$ is bounded from $L^{p}(\mathbb{R}, X)$ to $L^{p^{\prime}}(\mathbb{R}, X)$. Clearly, each Banach space has at least Fourier type $p=1$. Hilbert spaces have Fourier type $p=2$ by Plancherel's theorem. In fact, only Hilbert spaces have Fourier type 2. The space $X=L^{q}(\Omega)$ has Fourier type $p=\min \left\{q, q^{\prime}\right\}$. Moreover, uniformly convex Banach spaces have nontrivial Fourier type $p>1$. (See $[19$, p. 116] for references concerning these facts.) In particular, (1.5) implies (1.3) if $X$ is a Hilbert space. By means 
of an example it can be shown that the exponent $\beta$ in (1.5) is optimal (see [25, §4] or [19, Ex. 4.2.9]).

In Theorem 2.2 we establish a result on exponential dichotomy which is analogous to the Weis-Wrobel theorem. As in (1.5) we expect exponential estimates only for $x$ in a space $X_{\alpha}:=\mathcal{D}\left((w-A)^{\alpha}\right)$, correspondingly the dichotomy projection $P$ will only be defined on a subspace $\mathcal{D}(P)$ of $X$ containing $X_{\alpha}$. This leads us to the following concept which is weaker than the usual exponential dichotomy (where $\alpha=0$ and $\mathcal{D}(P)=X$ ).

Definition 1.1. Let $T(\cdot)$ be strongly continuous semigroup on a Banach space $X$ and $\alpha \geq 0$. We say that $T(\cdot)$ has an exponential $\alpha$-dichotomy if there is a closed projection $P$ on $X$ and constants $N, \delta>0$ such that

(a) $X_{\alpha} \hookrightarrow \mathcal{D}(P), T(t) \mathcal{D}(P) \subset \mathcal{D}(P), T(t) P x=P T(t) x, x \in \mathcal{D}(P)$,

(b) $T(t): \mathcal{N}(P) \rightarrow \mathcal{N}(P)$ has a bounded inverse, denoted by $T_{Q}(-t)$,

(c) $\|T(t) P x\| \leq N e^{-\delta t}\left\|(w-A)^{\alpha} x\right\|,\left\|T_{Q}(-t)(I-P) x\right\| \leq N e^{-\delta t}\left\|(w-A)^{\alpha} x\right\|$

for $t \geq 0$ and $x \in X_{\alpha}$, where we set $Q=I-P$. We call $\alpha$ and $\delta$ the regularity and decay exponent, respectively.

We note that unbounded splitting projections also occur in the study of bisectorial operators (see e.g. [17], [22]). However, this is a different situation insofar as semigroups generated by bisectorial operators are automatically analytic.

Our main Theorem 2.2 shows that $T(\cdot)$ has an exponential $\alpha$-dichotomy if $R(i \tau, A)$ exists and is uniformly bounded for $\tau \in \mathbb{R}$ and $\alpha>1 / p-1 / p^{\prime} \geq 0$, where $p \in[1,2]$ is the Fourier type of $X$. We can take $\alpha=1$ in the case of a nontrivial Fourier type $p>1$ (e.g., if $X$ is uniformly convex). In this case Definition 1.1(c) gives exponential estimates for $x \in \mathcal{D}(A)$, i.e., for classical solutions $u(t)=T(t) x$ of the Cauchy problem. Unfortunately, we do not quite obtain the exponent $\alpha=1 / p-1 / p^{\prime}$ from (1.5). For $P=I$, our theorem corresponds to the results of the paper [20], where (1.5) was shown for $\beta>1 / p-1 / p^{\prime}$. Then in [25] additional arguments were developed which allow one to pass to the equality $\beta=1 / p-1 / p^{\prime}$ in the regularity exponent. A different approach is contained in the proof of Theorem 4.5.2 of [19]. But it seems that these techniques do not work in the presence of an unbounded projection or if one deals with a spectral gap (as in our situation).

We are aware of only one result dealing with dichotomies in the setting of Theorem 2.2, namely Theorem 5.5 of the paper [7] by deLaubenfels and Latushkin. These authors obtain an exponential dichotomy on a Banach space $Z$ such that $\mathcal{D}\left(A^{2}\right) \subset Z \subset X$ assuming that $R(\lambda, A)$ is bounded on $i \mathbb{R}$. But it seems that $Z$ is hard to describe conveniently and that it is smaller than our $X_{\alpha}$. The approach of [7] is based on deLaubenfels' work on regularized functional calculi [6]. (Concerning these calculi we also refer to the 
recent contribution [10] and the fundamental work by McIntosh in e.g. [16] and [17].) We proceed in a different, rather direct and self-contained way: The contour integrals (2.8) and (2.2) below define operators on $X$ which turn out to be equal to $T(t) P(w-A)^{-\alpha}$ and $T_{Q}(-t)(I-P)(w-A)^{-\alpha}$ and to have the asserted properties. Our reasoning is inspired by methods from [12], [14], [20] and from the theory of functional calculi. But the verification of the exponential estimates in Definition 1.1(c) and the presence of an unbounded projection pose several new difficulties. We also note that we do not use the Weis-Wrobel theorem in our arguments (cf. Remark 2.3).

In Example 2.5 we study a parabolic partial differential equation in $L^{q}(\Omega)$ with a delay in the highest spatial derivatives, based on Theorem 2.2 and our work in [4]. We show that the exponential $\alpha$-dichotomy follows also in this case from a resolvent type estimate (where $\alpha>\left|1 / q-1 / q^{\prime}\right|$ ), and we give quite explicit sufficient conditions in a special case. Here pure spectral criteria for exponential dichotomy may fail (see [4, Ex. 5.8]).

In fact, our main Theorem 2.2 is stated in a somewhat more general way allowing for polynomial growth of $R(\lambda, A)$ on an open vertical strip around $i \mathbb{R}$. This extension only affects the value of the regularity exponent $\alpha$. If one merely assumes that $\|R(i \tau, A)\| \leq c\left(1+|\tau|^{\gamma}\right)$ for all $\tau \in \mathbb{R}$ and some $\gamma>0$, then it may happen that the spectrum approaches the imaginary axis at $\pm i \infty$. There are various examples arising from wave equations where $\sigma(A)$ belongs to the open left half-plane, the semigroup is bounded (thus $s(A)=0)$, and $T(t) x$ decays polynomially, but not exponentially as $t \rightarrow$ $\infty$ for $x \in \mathcal{D}(A)$ (see e.g. [1], [3], [15], and the references therein). This situation was investigated in detail in [3]. To our knowledge there are no papers treating the case that the generator spectrum $\sigma(A)$ approaches $i \mathbb{R}$ at $\pm i \infty$ from the left and the right. We address this point in the third section. In fact, the arguments of Section 2 can be modified in order to obtain again a closed projection $P$ with the properties from Definition 1.1 except for (c) where we now take $\alpha>\gamma+1 / p-1 / p^{\prime}$. In particular we have $X_{\alpha} \hookrightarrow \mathcal{D}(P)$. Extending the methods of Section 2, we can further show the polynomial decay on appropriate subspaces (see Theorem 3.1, (3.3), and (3.4)).

Notation and definitions. For Banach spaces $X$ and $Y$ the space of bounded linear operators is denoted by $\mathcal{B}(X, Y)$, where $\mathcal{B}(X):=\mathcal{B}(X, X)$. By $\mathcal{D}(B), \mathcal{N}(B), \mathcal{R}(B), \sigma(B), \varrho(B)$, we designate the domain, kernel, range, spectrum, and resolvent set of a linear operator $B$, respectively, and we set $R(\lambda, B)=(\lambda I-B)^{-1}=(\lambda-B)^{-1}$. The domain of a linear operator $B$ is always endowed with the graph norm of $B$. For $p \geq 1$ we set $p^{\prime}=p /(p-1)$ if $p>1$ and $p^{\prime}=\infty$ if $p=1$. The Fourier transform is defined by $\mathcal{F} f(\tau)=\int_{\mathbb{R}} e^{-i \tau t} f(t) d t, \tau \in \mathbb{R}$, for $f \in L^{1}(\mathbb{R}, X)$. We write $c=c(\alpha, \beta, \ldots)$ for a generic constant depending on the quantities $\alpha, \beta, \ldots$. 
Let $A$ be the generator of a $C_{0}$-semigroup $T(\cdot)=(T(t))_{t>0}$ on $X$. The spectral bound of $A$ is defined by $s(A)=\sup \{\operatorname{Re} \lambda: \lambda \in \sigma(A)\}$, and the growth bound by $\omega_{0}(A)=\inf \left\{w \in \mathbb{R}: \exists M:\|T(t)\| \leq M e^{w t}, t \geq 0\right\}$. We say that $T(\cdot)$ is exponentially stable if $\omega_{0}(A)<0$. Recall that $s(A) \leq$ $\omega_{0}(A)<\infty$. We fix a number $w>\omega_{0}(A)$ and define for $\alpha>0$ the fractional power

$$
(w-A)^{-\alpha}=\frac{1}{2 \pi i} \int_{\Gamma}(w-\lambda)^{-\alpha} R(\lambda, A) d \lambda
$$

where $\Gamma$ is a piecewise smooth path in the set $\left\{\lambda \in \mathbb{C}: \operatorname{Re} \lambda>\omega_{0}(A)\right.$, $\lambda \notin[w, \infty)\}$, running from $\infty e^{-i \phi}$ to $\infty e^{i \phi}$ for some $0<\phi<\pi / 2$. We further set $(w-A)^{0}=I$. The operators $(w-A)^{-\alpha}$ are injective, bounded, and satisfy the power law with respect to $\alpha$. In particular, $(w-A)^{-\alpha}$ has a closed inverse denoted by $(w-A)^{\alpha}$. The domain $X_{\alpha}:=\mathcal{D}\left((w-A)^{\alpha}\right)$ does not depend on the choice of $w>\omega_{0}(A)$. It is known that $X_{n}=\mathcal{D}\left(A^{n}\right)$ for $n \in \mathbb{N}$ (with equivalent norms) and that $X_{\beta} \hookrightarrow X_{\alpha} \hookrightarrow X$ for $\beta \geq \alpha \geq 0$, where "œ" designates a continuous embedding (which is also dense in our case). Since $(w-A)^{-\alpha} T(t)=T(t)(w-A)^{-\alpha}$ it is easy to see that the restriction $A_{\alpha}: X_{1+\alpha} \rightarrow X_{\alpha}$ of $A$ generates the semigroup in $X_{\alpha}$ given by the restrictions of $T(t)$ to $X_{\alpha}$. Moreover, $R\left(\lambda, A_{\alpha}\right)$ is the restriction of $R(\lambda, A)$ to $X_{\alpha}$ for $\lambda \in \varrho\left(A_{\alpha}\right)=\varrho(A)$. We refer to [2] or [8] for proofs of these facts.

2. Exponential dichotomy. Before presenting our main theorem we state a standard lemma on closed projections. The proof is given for the reader's convenience. Here a "closed projection" $P$ on $X$ is a closed linear operator such that $P \mathcal{D}(P) \subset \mathcal{D}(P)$ and $P x=P^{2} x$ for $x \in \mathcal{D}(P)$. Throughout we set $Q=I-P$ with $\mathcal{D}(Q)=\mathcal{D}(P)$.

Lemma 2.1. If $P$ is a closed projection on a Banach space $X$, then the spaces $\mathcal{N}(P)=\mathcal{R}(Q)$ and $\mathcal{R}(P)=\mathcal{N}(Q)$ are closed in $X$ and in $\mathcal{D}(P)$. Moreover, $\mathcal{D}(P)=\mathcal{N}(P) \oplus \mathcal{R}(P)$.

Proof. We first observe that $Q$ is also a closed projection on $X$ so that the kernels $\mathcal{N}(P)$ and $\mathcal{N}(Q)$ are closed in $X$ and $\mathcal{D}(P)$. It is clear that $\mathcal{N}(Q) \subset \mathcal{R}(P)$. Conversely, a vector $y=P x$ belongs to $\mathcal{D}(P)=\mathcal{D}(Q)$ and $Q y=P x-P^{2} x=0$. As a result, $\mathcal{N}(P)=\mathcal{R}(Q)$ and $\mathcal{N}(Q)=\mathcal{R}(I-Q)=$ $\mathcal{R}(P)$. To show that $\mathcal{D}(P)=\mathcal{N}(P) \oplus \mathcal{R}(P)$, we take $x \in \mathcal{N}(P) \cap \mathcal{R}(P)$. Then $x=P x=0$. Further, $x \in \mathcal{D}(P)$ can be decomposed into $x=P x+(I-P) x \in$ $\mathcal{R}(P)+\mathcal{R}(Q)=\mathcal{R}(P)+\mathcal{N}(P)$.

Theorem 2.2. Let $A$ be the generator of a $C_{0}$-semigroup $T(\cdot)$ on a Banach space $X$ with Fourier type $p \in[1,2]$. 
(1) Suppose that either

(a) $i \mathbb{R} \subset \varrho(A)$ and $\|R(i \tau, A)\| \leq C$ for $\tau \in \mathbb{R}$ and a constant $C>0$, or

(b) $\{\lambda \in \mathbb{C}:|\operatorname{Re} \lambda| \leq \delta\} \subset \varrho(A)$ and $\|R(\lambda, A)\| \leq \widetilde{C}\left(1+|\lambda|^{\gamma}\right)$ for $|\operatorname{Re} \lambda| \leq \delta$ and some constants $\widetilde{C}, \delta, \gamma>0$.

In case (a), we set $\gamma=0$ and take $\delta \in(0,1 / C)$. Let $\alpha>1 / p-1 / p^{\prime}+\gamma$. Then $T(\cdot)$ has an exponential $\alpha$-dichotomy with decay exponent $\delta$ and a constant $N=N(\alpha)$.

(2) Conversely, if $T(\cdot)$ has an exponential $\alpha$-dichotomy with decay exponent $\delta_{0}>0$, then (b) holds with $\gamma=\alpha$ and every $\delta \in\left(0, \delta_{0}\right)$.

Proof. To show the second part of the theorem, assume that $T(\cdot)$ has an exponential $\alpha$-dichotomy with decay exponent $\delta_{0}>0$, and let $x \in X_{\alpha}$. Then the operator

$$
R_{\lambda} x=\int_{0}^{\infty} e^{-\lambda t} T(t) P x d t-\int_{0}^{\infty} e^{\lambda t} T_{Q}(-t) Q x d t
$$

maps $X_{\alpha}$ into $X$ and is uniformly bounded for $|\operatorname{Re} \lambda| \leq \delta$ and a fixed $0<\delta<\delta_{0}$. It is then straightforward to check that $R_{\lambda} x \in \mathcal{D}(A)$ and $(\lambda-A) R_{\lambda} x=x$ and that $R_{\lambda}(\lambda-A) y=y$ for $y \in X_{1+\alpha}$. Hence, $\lambda \in \varrho\left(A_{\alpha}\right)$ $=\varrho(A)$, where $A_{\alpha}$ is the part of $A$ in $X_{\alpha}$, and $R(\lambda, A)$ is an extension of $R_{\lambda}$. Property (b) now follows from [14, Lem. 3.2] and the uniform boundedness of $R(\lambda, A)(w-A)^{-\alpha}=R_{\lambda}(w-A)^{-\alpha}$ for $|\operatorname{Re} \lambda| \leq \delta$.

We prove the first part of Theorem 2.2 in four steps. We first observe that assumption (a) implies (b) with $\gamma=0$ and some $0<\delta<1 / C$ by a standard perturbation argument. We fix numbers $\alpha>1 / p-1 / p^{\prime}+\gamma \geq \gamma \geq 0$, $0 \leq a \leq \delta$, and $\max \left\{\delta, \omega_{0}(A)\right\}<\widetilde{w}<w$. Hence, $\|R(\lambda, A)\| \leq c$ for $\operatorname{Re} \lambda \geq \widetilde{w}$.

STEP 1: First part of the construction of $T_{Q}(-t) Q$. We define the path $\Gamma_{Q}=(\widetilde{w}+[-i \infty,+i \infty]) \cup(a+[+i \infty,-i \infty])$ which is oriented counterclockwise. Let $x \in \mathcal{D}(A)$ and $t \geq 0$. Since

$$
\begin{aligned}
R(\lambda, A) x & =(w-\lambda)^{-1}(R(\lambda, A)-R(w, A))(w-A) x \\
& =\mathcal{O}\left(|\lambda|^{\gamma-1}\right), \quad|\operatorname{Im} \lambda| \rightarrow \infty,
\end{aligned}
$$

for $|\operatorname{Re} \lambda| \leq \delta$ or $\operatorname{Re} \lambda=\widetilde{w}$ the integral

$$
G_{Q}(t) x=\frac{1}{2 \pi i} \int_{\Gamma_{Q}} e^{-\lambda t}(w-\lambda)^{-\alpha} R(\lambda, A) x d \lambda
$$

converges absolutely. Consider the counter-clockwise oriented rectangular path $\Gamma_{n}$ with vertices $\widetilde{w} \pm i n$ and $a \pm i n$. For $s \in[0, t]$ we then obtain 


$$
\begin{aligned}
& T(s) G_{Q}(t) x=G_{Q}(t) T(s) x \\
& \quad=\frac{1}{2 \pi i} \int_{\Gamma_{Q}} e^{-\lambda(t-s)}(w-\lambda)^{-\alpha}\left[R(\lambda, A) x-\int_{0}^{s} e^{-\lambda r} T(r) x d r\right] d \lambda \\
& \quad=G_{Q}(t-s) x-\lim _{n \rightarrow \infty} \frac{1}{2 \pi i} \int_{\Gamma_{n}} e^{-\lambda(t-s)}(w-\lambda)^{-\alpha} \int_{0}^{s} e^{-\lambda r} T(r) x d r d \lambda \\
& =G_{Q}(t-s) x,
\end{aligned}
$$

where we have used Cauchy's theorem and

$$
\int_{0}^{s} e^{-\lambda r} T(r) x d r=\left(e^{-\lambda s} T(s)-I\right) R(\lambda, A) x=\mathcal{O}\left(|\lambda|^{\gamma-1}\right)
$$

on $\Gamma_{Q}$ as $|\operatorname{Im} \lambda| \rightarrow \infty$ due to (2.1). We define

$$
\begin{aligned}
& \widetilde{Q} x:=G_{Q}(0) x \\
& =\frac{1}{2 \pi i} \int_{+i \infty}^{-i \infty}(w-\lambda)^{-\alpha} R(\lambda, A) x d \lambda+\frac{1}{2 \pi i} \int_{\widetilde{w}-i \infty}^{\widetilde{w}+i \infty}(w-\lambda)^{-\alpha} R(\lambda, A) x d \lambda \\
& =-\frac{1}{2 \pi i} \int_{-i \infty}^{+i \infty}(w-\lambda)^{-\alpha} R(\lambda, A) x d \lambda+(w-A)^{-\alpha} x
\end{aligned}
$$

for $x \in \mathcal{D}(A)$. The last equality follows from a standard deformation of the path $[\widetilde{w}-i \infty, \widetilde{w}+i \infty]$ to the path $\Gamma$ from (1.6).

Observe that the function $t \mapsto T_{\widetilde{w}}(t):=e^{-\widetilde{w} t} T(t)$ belongs to $L^{2}\left(\mathbb{R}_{+}, \mathcal{B}(X)\right)$. Since $R(\widetilde{w}+i \tau, A) x=\left(\mathcal{F} T_{\widetilde{w}}(\cdot) x\right)(\tau)$ and $p$ is the Fourier type of $X$, we obtain $R(\widetilde{w}+i \cdot, A) x \in L^{p^{\prime}}(\mathbb{R}, X)$ with $p^{\prime}$-norm less than $c\|x\|$. The resolvent equation further yields

$$
\begin{aligned}
\|R(a+i \tau, A) x\| & =\|[I+(\widetilde{w}-a) R(a+i \tau, A)] R(\widetilde{w}+i \tau, A) x\| \\
& \leq c(1+|\tau|)^{\gamma}\|R(\widetilde{w}+i \tau, A) x\|
\end{aligned}
$$

for $\tau \in \mathbb{R}$. We set

$$
f(\lambda)=(w-\lambda)^{-\alpha} R(\lambda, A) x= \begin{cases}f_{1}(\tau), & \lambda=a+i \tau, \tau \in \mathbb{R} \\ f_{2}(\tau), & \lambda=\widetilde{w}+i \tau, \tau \in \mathbb{R} .\end{cases}
$$

Let $p \in(1,2]$. Then Hölder's inequality, estimate (2.5), and the inequality $(-\alpha+\gamma) p p^{\prime}\left(p^{\prime}-p\right)^{-1}<-1$ yield

$$
\begin{aligned}
& \int_{\mathbb{R}}\left\|f_{1}(\tau)\right\|^{p} d \tau \\
\leq & c\left[\int_{\mathbb{R}}\|R(\widetilde{w}+i \tau, A) x\|^{p^{\prime}} d \tau\right]^{p / p^{\prime}}\left[\int_{\mathbb{R}}(1+|\tau|)^{(-\alpha+\gamma) p p^{\prime} /\left(p^{\prime}-p\right)} d \tau\right]^{\left(p^{\prime}-p\right) / p^{\prime}} \\
\leq & c(\alpha)\|x\|^{p} .
\end{aligned}
$$


The function $f_{2}$ can be estimated similarly. Thus the identity

$$
\begin{aligned}
2 \pi e^{a t} G_{Q}(t) x & =\frac{1}{i} \int_{\Gamma_{Q}} e^{(a-\lambda) t}(w-\lambda)^{-\alpha} R(\lambda, A) x d \lambda \\
& =-\left(\mathcal{F} f_{1}\right)(t)+e^{(a-\widetilde{w}) t}\left(\mathcal{F} f_{2}\right)(t),
\end{aligned}
$$

inequality (2.6), and the Fourier type of $X$ imply that

$$
\int_{0}^{\infty}\left\|e^{a t} G_{Q}(t) x\right\|^{p^{\prime}} d t \leq c\left(\left\|f_{1}\right\|_{p}^{p^{\prime}}+\left\|f_{2}\right\|_{p}^{p^{\prime}}\right) \leq c(\alpha)\|x\|^{p^{\prime}} .
$$

From this estimate and formula (2.3) we then deduce

$$
\begin{aligned}
\left\|e^{a r} G_{Q}(r) x\right\|^{p^{\prime}} & \leq \int_{0}^{1} e^{p^{\prime} a(r+s)}\|T(s)\|^{p^{\prime}}\left\|G_{Q}(r+s) x\right\|^{p^{\prime}} d s \\
& \leq c \int_{r}^{r+1}\left\|e^{a t} G_{Q}(t) x\right\|^{p^{\prime}} d t \leq c(\alpha)\|x\|^{p^{\prime}}
\end{aligned}
$$

for $r \geq 0$ and $x \in \mathcal{D}(A)$. Therefore we can extend $G_{Q}(t)$ to a bounded operator on $X$ denoted by the same symbol and satisfying

$$
\left\|G_{Q}(t)\right\| \leq c(\alpha) e^{-\delta t}, \quad t \geq 0
$$

where we let $a=\delta$. In particular, the extension $\widetilde{Q}: X \rightarrow X$ is bounded. The same results hold for $p=1$ by an analogous, but simpler argument.

STEP 2: Construction of $T(t) P$. We define for $x \in \mathcal{D}(A)$ and $t \geq 0$ the operator

$$
G_{P}(t) x=\frac{1}{2 \pi i} \int_{-a-i \infty}^{-a+i \infty} e^{\lambda t}(w-\lambda)^{-\alpha} R(\lambda, A) x d \lambda .
$$

Again (2.1) shows that the integral converges absolutely. If $t=0$, we set

$$
\widetilde{P} x:=G_{P}(0) x=\frac{1}{2 \pi i} \int_{-i \infty}^{+i \infty}(w-\lambda)^{-\alpha} R(\lambda, A) x d \lambda
$$

(after shifting $a$ to 0 ). Thus (2.4) implies

$$
\widetilde{P} x+\widetilde{Q} x=(w-A)^{-\alpha} x
$$

for $x \in \mathcal{D}(A)$. There exists a bounded extension $\widetilde{P}: X \rightarrow X$ due to (2.7) and (2.10). For $x \in \mathcal{D}\left(A^{2}\right)$ and $t \geq 0$ we further compute

$$
\begin{aligned}
G_{P}(t) x & =\frac{1}{2 \pi i} \int_{-a-i \infty}^{-a+i \infty} e^{\lambda t}(w-\lambda)^{-\alpha-1}(R(\lambda, A)-R(w, A))(w-A) x d \lambda \\
& =\frac{1}{2 \pi i} \int_{-a-i \infty}^{-a+i \infty} e^{\lambda t}(w-\lambda)^{-\alpha-1} R(\lambda, A)(w-A) x d \lambda,
\end{aligned}
$$


using the resolvent equation and

$$
\frac{1}{2 \pi i} \int_{-a-i \infty}^{-a+i \infty} e^{\lambda t}(w-\lambda)^{-\alpha-1} d \lambda=0
$$

(which can be shown by applying Cauchy's theorem to the triangular path with vertices $-a-i n,-a+i n,-n)$. Because of the identity

$$
\begin{aligned}
& \frac{d}{d t} e^{\lambda t}(w-\lambda)^{-\alpha-1} R(\lambda, A)(w-A) x \\
& \quad=e^{\lambda t}(w-\lambda)^{-\alpha-1} R(\lambda, A)(w-A) A x+e^{\lambda t}(w-\lambda)^{-\alpha-1}(w-A) x,
\end{aligned}
$$

equation (2.11), and the closedness of $A$, the function $G_{P}(\cdot) x$ is continuously differentiable in $X, G_{P}(t) \mathcal{D}\left(A^{2}\right) \subset \mathcal{D}(A)$, and

$$
\frac{d}{d t} G_{P}(t) x=G_{P}(t) A x=A G_{P}(t) x, \quad t \geq 0
$$

for $x \in \mathcal{D}\left(A^{2}\right)$. Therefore the uniqueness of the Cauchy problem corresponding to $A$ yields

$$
G_{P}(t) x=T(t) \widetilde{P} x, \quad t \geq 0,
$$

at first for $x \in \mathcal{D}\left(A^{2}\right)$. Since $T(t) \widetilde{P}$ is bounded, we can extend $G_{P}(t)$ to a bounded operator (denoted by the same symbol) which satisfies (2.13) for all $x \in X$. We have $A \widetilde{P} x=\widetilde{P} A x$ on $\mathcal{D}\left(A^{2}\right)$ by $(2.12)$, so that

$$
\widetilde{P} \mathcal{D}(A) \subset \mathcal{D}(A), \quad A \widetilde{P} x=\widetilde{P} A x \quad \text { for } x \in \mathcal{D}(A),
$$

and hence

$$
T(t) \widetilde{P}=\widetilde{P} T(t), \quad t \geq 0 .
$$

Let $p \in(1,2]$. As in the first step one shows that

$$
\int_{0}^{\infty}\left\|e^{a t} T(t) \widetilde{P} x\right\|^{p^{\prime}} d t \leq c(\alpha)\|x\|^{p^{\prime}}
$$

for $x \in \mathcal{D}(A)$. For $r \geq 1$, this estimate implies that

$$
\left\|e^{a r} T(r) \widetilde{P} x\right\|^{p^{\prime}} \leq \int_{0}^{1}\left\|e^{a s} T(s)\right\|^{p^{\prime}}\left\|e^{a(r-s)} T(r-s) \widetilde{P} x\right\|^{p^{\prime}} d s \leq c(\alpha)\|x\|^{p^{\prime}} .
$$

Taking $a=\delta$, we have shown that

$$
\|T(t) \widetilde{P}\| \leq c(\alpha) e^{-\delta t}, \quad t \geq 0
$$

Again, this inequality still holds for $p=1$ due to a similar argument.

STEP 3: The projection $P$. We define the bounded operator

$$
P=\widetilde{P}(w-A)^{\alpha}: X_{\alpha} \rightarrow X \text {. }
$$


Observe that the first part of Definition 1.1(c) now follows from (2.15). For $x \in X_{1+\alpha}$, formula (2.9) and the closedness of the fractional power further imply that $\widetilde{P} X_{1+\alpha} \subset X_{\alpha}$ and

$$
P x=\frac{1}{2 \pi i} \int_{-i \infty}^{+i \infty}(w-\lambda)^{-\alpha} R(\lambda, A)(w-A)^{\alpha} x d \lambda=(w-A)^{\alpha} \widetilde{P} x .
$$

Hence, $\widetilde{P} X_{\alpha} \subset X_{\alpha}$ and $P=(w-A)^{\alpha} \widetilde{P}$ on $X_{\alpha}$. Since $(w-A)^{\alpha}$ is closed, the operator $P$ possesses a closure (denoted by $(P, \mathcal{D}(P))$ ) in $X$. We further obtain $X_{\alpha} \hookrightarrow \mathcal{D}(P), \widetilde{P} \mathcal{D}(P) \subset X_{\alpha}, P=(w-A)^{\alpha} \widetilde{P}$ on $\mathcal{D}(P)$, and therefore

$$
\widetilde{P}=P(w-A)^{-\alpha}, \quad \widetilde{P} x=(w-A)^{-\alpha} P x \quad \text { for } x \in \mathcal{D}(P) .
$$

We set $Q=I-P$. Then the identities (2.10) and (2.17) yield

$$
\widetilde{Q}=Q(w-A)^{-\alpha}, \quad \widetilde{Q} x=(w-A)^{-\alpha} Q x \quad \text { for } x \in \mathcal{D}(P) .
$$

We are going to show that $P$ is a projection. For $x \in \mathcal{D}\left(A^{2}\right)$ and $a>0$ we calculate

$$
\begin{aligned}
\widetilde{P}^{2} x= & \frac{1}{2 \pi i} \int_{-a-i \infty}^{-a+i \infty} \frac{1}{2 \pi i} \int_{-i \infty}^{+i \infty}(w-\lambda)^{-\alpha}(w-\mu)^{-\alpha} R(\lambda, A) R(\mu, A) x d \mu d \lambda \\
= & \frac{1}{2 \pi i} \int_{-a-i \infty}^{-a+i \infty}(w-\lambda)^{-\alpha} \frac{1}{2 \pi i} \int_{-i \infty}^{+i \infty} \frac{(w-\mu)^{-\alpha}}{\mu-\lambda} d \mu R(\lambda, A) x d \lambda \\
& +\frac{1}{2 \pi i} \int_{-i \infty}^{+i \infty}(w-\mu)^{-\alpha} \frac{1}{2 \pi i} \int_{-a-i \infty}^{-a+i \infty} \frac{(w-\lambda)^{-\alpha}}{\lambda-\mu} d \lambda R(\mu, A) x d \mu
\end{aligned}
$$

using the resolvent equation and Fubini's theorem. The integration path of the scalar $\lambda$-integral in the last line can be closed in the left half-plane where the integrand is holomorphic. Thus the second summand on the right hand side is equal to 0 . In the scalar $\mu$-integral in the first summand, we use the triangular path with vertices $-i n, i n,-n$ for large $n \in \mathbb{N}$ such that the (fixed) number $\lambda$ belongs to the interior of the triangle. Then Cauchy's integral formula yields

$$
\begin{aligned}
\widetilde{P}^{2} x & =\frac{1}{2 \pi i} \int_{-a-i \infty}^{-a+i \infty}(w-\lambda)^{-2 \alpha} R(\lambda, A) x d \lambda \\
& =\frac{1}{2 \pi i} \int_{-i \infty}^{+i \infty}(w-\lambda)^{-2 \alpha} R(\lambda, A) x d \lambda
\end{aligned}
$$


On the other hand, by similar arguments we deduce

$$
\begin{aligned}
\widetilde{P}(w-A)^{-\alpha} x \\
=\frac{1}{2 \pi i} \int_{-i \infty}^{+i \infty} \frac{1}{2 \pi i} \int_{\Gamma}(w-\lambda)^{-\alpha}(w-\mu)^{-\alpha} R(\lambda, A) R(\mu, A) x d \mu d \lambda \\
=\frac{1}{2 \pi i} \int_{-i \infty}^{+i \infty}(w-\lambda)^{-\alpha} \frac{1}{2 \pi i} \int_{\Gamma} \frac{(w-\mu)^{-\alpha}}{\mu-\lambda} d \mu R(\lambda, A) x d \lambda \\
\quad+\frac{1}{2 \pi i} \int_{\Gamma}(w-\mu)^{-\alpha} \frac{1}{2 \pi i} \int_{-i \infty}^{+i \infty} \frac{(w-\lambda)^{-\alpha}}{\lambda-\mu} d \lambda R(\mu, A) x d \mu \\
=\frac{1}{2 \pi i} \int_{-i \infty}^{+i \infty}(w-\lambda)^{-2 \alpha} R(\lambda, A) x d \lambda .
\end{aligned}
$$

In the first summand of the middle equation we have deformed a bounded part of the path $\Gamma$ from (1.6) to a path in $\{\mu: \operatorname{Re} \mu<w\}$ around the (fixed) number $\lambda \in i \mathbb{R}$. By approximation, the identities (2.20) and (2.21) can be extended to all $x \in X$. These two equations and (2.17) then yield

$$
\widetilde{P}^{2}=\widetilde{P}(w-A)^{-\alpha}=(w-A)^{-\alpha} \widetilde{P} .
$$

Hence, $\mathcal{R}\left(\widetilde{P}^{2}\right) \subset X_{\alpha}$ and $\widetilde{P}=(w-A)^{\alpha} \widetilde{P}^{2}$. Using again formula $(2.17)$ and the closedness of $P$, we conclude that $\widetilde{P} X \subset \mathcal{D}(P)$ and $\widetilde{P}=P \widetilde{P}$. This fact leads to $P=P^{2}$ on $X_{\alpha}$, due to (2.17). As a result, $P$ is a projection. Combining (2.14) and (2.16) we further deduce $T(t) P=P T(t)$ on $X_{\alpha}$, so that $T(t) \mathcal{D}(P) \subset \mathcal{D}(P)$ and $T(t) P=P T(t)$ on $\mathcal{D}(P)$ by approximation.

STEP 4: Second part of the construction of $T_{Q}(-t) Q$. Formula (2.2) implies that $G_{Q}(t) X_{1+\alpha} \subset X_{\alpha}$ and $G_{Q}(t)(w-A)^{\alpha} x=(w-A)^{\alpha} G_{Q}(t) x$ for $x \in X_{1+\alpha}$ and $t \geq 0$. Thus $G_{Q}(t) X_{\alpha} \subset X_{\alpha}$ and $G_{Q}(t)$ commutes with $(w-A)^{\alpha}$. For $x \in X_{1+\alpha}$ and $t \geq 0$, the equalities (2.3) and (2.18) then show that

$$
\begin{aligned}
& (w-A)^{\alpha} G_{Q}(t) T(t) x=(w-A)^{\alpha} \widetilde{Q} x=Q x, \\
& T(t)(w-A)^{\alpha} G_{Q}(t) x=(w-A)^{\alpha} \widetilde{Q} x=Q x .
\end{aligned}
$$

One can extend (2.22) to $\mathcal{D}(P)$ and (2.23) to $X_{\alpha}$. Therefore the restriction $T_{Q}(t)$ of $T(t)$ to $\mathcal{N}(P)=\mathcal{R}(Q)$ is injective; its (closed) inverse is denoted by $T_{Q}(-t)$. Then $(2.23)$ and an approximation argument yield

$$
G_{Q}(t) x=(w-A)^{-\alpha} T_{Q}(-t) Q x
$$

for $x \in \mathcal{D}(P)$. Inserting this identity into (2.22), we see that $T(t)$ : $\mathcal{N}(P) \rightarrow \mathcal{N}(P)$ is bijective, and its inverse $T_{Q}(-t): \mathcal{N}(P) \rightarrow \mathcal{N}(P)$ is 
bounded by the closed graph theorem. Moreover,

$$
T_{Q}(-t) Q x=G_{Q}(t)(w-A)^{\alpha} x, \quad x \in X_{\alpha},
$$

and the second part of Definition 1.1(c) follows from (2.7).

Remark 2.3. Suppose that the assumptions of Theorem 2.2 hold for $\gamma=0$. Let $T_{s}(t)$ be the restriction of $T(t)$ to $X_{s}:=\mathcal{R}(P)$. Then the generator $A_{s}$ of this semigroup is the restriction of $A$ to $X_{s}$. Definition 1.1(c) implies that $R\left(\lambda, A_{s}\right)\left(w-A_{s}\right)^{-\alpha}$ (initially defined for $\operatorname{Re} \lambda>\omega_{0}\left(A_{s}\right)$ ) has a bounded extension $F(\lambda)$ to $\operatorname{Re} \lambda \geq-\delta / 2$. This means that the spectral bound of the part of $A_{s}$ in $\mathcal{D}\left(\left(w-A_{s}\right)^{\alpha}\right)$ is less than $-\delta / 2$, and hence $s\left(A_{s}\right) \leq-\delta / 2<0$. The boundedness of $F(\lambda)=R\left(\lambda, A_{s}\right)\left(w-A_{s}\right)^{-\alpha}$ further yields $\left\|R\left(\lambda, A_{s}\right)\right\| \leq$ $c\left(1+|\lambda|^{\alpha}\right)$ for $\operatorname{Re} \lambda \geq-\delta / 2$ by [14, Lem. 3.2]. On the other hand, $R\left(\lambda, A_{s}\right)$ is the restriction of $R(\lambda, A)$ for $\lambda \in i \mathbb{R}$, and thus uniformly bounded on $i \mathbb{R}$ by assumption (a). The Phragmén-Lindelöf principle then implies that $R\left(\lambda, A_{s}\right)$ is uniformly bounded for $\operatorname{Re} \lambda \geq 0$. The Weis-Wrobel result (1.5) now shows that

$$
\|T(t) x\| \leq N e^{-\delta t}\left\|(w-A)^{\beta} x\right\|, \quad t \geq 0,
$$

for $\beta=1 / p-1 / p^{\prime}$ and $x \in \mathcal{R}(P) \cap X_{\beta}$. Similar arguments work for the restriction $-A_{u}$ of $-A$ to $X_{u}=\mathcal{R}(Q)$ which generates the semigroup $T_{Q}(-t)$, $t \geq 0$, on $X_{u}$. Hence

$$
\left\|T_{Q}(-t) x\right\| \leq N e^{-\delta t}\left\|(w-A)^{\beta} x\right\|, \quad t \geq 0,
$$

for $x \in \mathcal{R}(Q) \cap X_{\beta}$. If one wants to derive an estimate for $T(t) P$ as in our Definition 1.1, then one obtains

$$
\|T(t) P x\| \leq N e^{-\delta t}\left\|(w-A)^{\beta} P x\right\| \leq c e^{-\delta t}\left\|(w-A)^{\beta+\alpha} x\right\|
$$

for $x \in X_{\beta+\alpha}$ with $\alpha>1 / p-1 / p^{\prime}$ (and analogously for $T_{Q}(-t) Q$ ). Observe that one looses $\beta=1 / p-1 / p^{\prime}$ in the regularity exponent, and it is thus necessary to estimate the products $T(t) P$ and $T_{Q}(-t) Q$ in order to prove Theorem 2.2.

REMARK 2.4. Suppose that the assumptions of Theorem 2.2 hold. Arguing as in (2.21), one can verify that

$$
\widetilde{P}(w-A)^{-\beta} x=(w-A)^{-\beta} \widetilde{P} x=\frac{1}{2 \pi i} \int_{-i \infty}^{+i \infty}(w-\lambda)^{-\alpha-\beta} R(\lambda, A) x d \lambda
$$

for $\beta>0$ and $x \in \mathcal{D}(A)$. This identity implies in particular that the projection $P$ in Theorem 2.2 does not depend on the choice of $\alpha>1 / p-1 / p^{\prime}+\gamma$.

In the next example we extend Theorem 4.4(a) and Proposition 5.7 from [4] from an $L^{2}$ to an $L^{q}$ setting. Due to the lack of a Weis-Wrobel type result for dichotomies, we were forced to restrict ourselves to a Hilbert space setting in [4]. 
ExAmple 2.5. Let $A=\Delta$ be the Dirichlet Laplacian with $\mathcal{D}(A)=$ $W^{2, q}(\Omega) \cap W_{0}^{1, q}(\Omega)=: X_{1}$ on $X=L^{q}(\Omega)$ for $1<q<\infty$ and a bounded domain $\Omega$ with a $C^{2}$ boundary. (In fact, one can replace $A$ by more general generators of analytic semigroups; see Hypothesis $(\mathrm{H})$ and Proposition 5.7 of [4] for the details.) Let $r>0$ and $B:[-r, 0] \rightarrow \mathcal{B}\left(X_{1}, X\right)$ be of bounded variation $d b$ such that $d b([-t, 0]) \rightarrow 0$ as $t \searrow 0$. We define the "history function" $u_{t}(\theta)=u(t+\theta)$ for $u:[-r, \infty) \rightarrow X, t \geq 0$, and $\theta \in[-r, 0]$. Let $p \in\left[\min \left\{q, q^{\prime}\right\}, \max \left\{q, q^{\prime}\right\}\right]$. For $\varphi \in W^{1, p}\left([-r, 0], X_{1}\right)$ we study the retarded problem

$$
\begin{aligned}
u^{\prime}(t) & =A u(t)+\int_{-r}^{0} d B(\theta) u(t+\theta), \quad t \geq 0, \\
u(t) & =\varphi(t), \quad t \in[-r, 0] .
\end{aligned}
$$

This problem can be solved using Theorems 7.4-7.6 of [21] which address even more general equations. In [4] we have developed a semigroup approach which gives an alternative proof of a part of these theorems. We need the real interpolation space $Y:=\left(X, X_{1}\right)_{1-1 / p, p}$. The product space $\mathcal{X}=Y \times$ $L^{p}([-r, 0], Y)$ has Fourier type $\min \left\{q, q^{\prime}\right\}$ (see e.g. the proof of $[4$, Thm. 4.5]). There is a unique solution $u \in C^{1}\left(\mathbb{R}_{+}, Y\right) \cap W_{\text {loc }}^{1, p}\left(\mathbb{R}_{+}, X_{1}\right)$ of (2.24). There exists a $C_{0}$-semigroup $\mathcal{T}(\cdot)$ on $\mathcal{X}$ satisfying

$$
\left(\begin{array}{c}
u(t) \\
u_{t}
\end{array}\right)=\mathcal{T}(t)\left(\begin{array}{c}
\varphi(0) \\
\varphi
\end{array}\right), \quad t \geq 0
$$

which is generated by

$$
\begin{aligned}
\mathcal{A} & =\left(\begin{array}{cc}
A & L \\
0 & d / d \theta
\end{array}\right), \\
D(\mathcal{A}) & =\left\{\left(\begin{array}{l}
x \\
\varphi
\end{array}\right) \in X_{1} \times W^{1, p}\left([-r, 0], X_{1}\right): \varphi(0)=x, L \varphi+A x \in Y\right\},
\end{aligned}
$$

where $L \varphi:=\int_{-r}^{0} d B(\theta) \varphi(\theta)$ for $\varphi \in W^{1, p}\left([-r, 0], X_{1}\right)$ (see [4, Thm. 3.6]). In certain cases this semigroup violates the spectral mapping theorem (see [4, Ex. 5.8]). Set $L_{\lambda} x=L\left(e_{\lambda} x\right)$ for $\lambda \in \mathbb{C}, x \in X_{1}$, and $e_{\lambda}(\theta)=e^{\lambda \theta}$, $\theta \in[-r, 0]$. By Proposition 4.3 of $[4], \lambda \in \mathbb{C}$ belongs to $\varrho(\mathcal{A})$ if and only if the operator $H(\lambda)=\left(\lambda-A-L_{\lambda}\right)^{-1}$ exists in $\mathcal{B}\left(X, X_{1}\right)$. One further has $\|R(\lambda, \mathcal{A})\|_{\mathcal{B}(\mathcal{X})} \leq c(a)\|H(\lambda)\|_{\mathcal{B}\left(X, X_{1}\right)}$ for $\lambda \in \varrho(\mathcal{A})$ with $\operatorname{Re} \lambda \geq a$. Thus Theorem 2.2 immediately implies that

$$
\begin{aligned}
& \sup _{\tau \in \mathbb{R}}\|H(i \tau)\|_{\mathcal{B}\left(X, X_{1}\right)}<\infty \\
& \quad \Rightarrow \mathcal{T}(\cdot) \text { has an exponential } \alpha \text {-dichotomy for } \alpha>\left|1 / q-1 / q^{\prime}\right| .
\end{aligned}
$$

Observe that the dichotomy of $\mathcal{T}(\cdot)$ means a splitting of both the solutions $u(t)$ and their history functions $u_{t}$ with decay estimates in the norm of $\mathcal{X}$. 
To obtain a more explicit condition, suppose in addition that $B(\theta)=$ $\eta(\theta) A$, where $\eta \in \mathrm{BV}([-r, 0], \mathbb{C})$. We set

$$
\widehat{d \eta}(\lambda)=\int_{-r}^{0} e^{\lambda \theta} d \eta(\theta), \quad \lambda \in \mathbb{C} .
$$

Then $H(i \tau): X \rightarrow X_{1}$ exists and is uniformly bounded for $\tau \in \mathbb{R}$ provided that

$$
\overline{1+\widehat{d \eta}(i \mathbb{R})} \subset\{\lambda \in \mathbb{C} \backslash\{0\}:|\arg \lambda|<\psi \text { or }|\arg (-\lambda)|<\psi\}
$$

for some $0<\psi<\pi / 2$ (see the proof of Proposition 5.7 of [4]). In this case we thus obtain an exponential $\alpha$-dichotomy.

3. Polynomial dichotomy. In this section we assume that $A$ is the generator of a semigroup $T(\cdot)$ on a Banach space $X$ such that $i \mathbb{R} \subset \varrho(A)$ and $\|R(i \tau, A)\| \leq \widehat{C}\left(1+|\tau|^{\gamma}\right)$ for $\tau \in \mathbb{R}$ and some $\gamma>0$. We take again $\alpha>\gamma+1 / p-1 / p^{\prime}$ where $p \in[1,2]$ is the Fourier type of $X$. We want to repeat the reasoning in Steps 1-4 of the proof of Theorem 2.2 with $a=\delta=0$. This can be done literally with the exception of (2.19). Here we have to replace the path $-a+i \mathbb{R}$ by the path $\Gamma^{\prime}$ given by

$$
\lambda=i \tau-\left[2 \widehat{C}\left(1+|\tau|^{\gamma}\right)\right]^{-1}, \quad \tau \in \mathbb{R} .
$$

Standard perturbation arguments show that $\Gamma^{\prime} \subset \varrho(A)$ and

$$
\|R(\lambda, A)\| \leq 2 \widehat{C}\left(1+|\operatorname{Im} \lambda|^{\gamma}\right) \leq c\left(1+|\lambda|^{\gamma}\right), \quad \lambda \in \Gamma^{\prime} .
$$

The modification of (2.19) can be justified using Cauchy's integral theorem. Then one verifies (2.20) as before. Thus we have constructed a closed projection $P$ on $X$ such that $X_{\alpha} \hookrightarrow \mathcal{D}(P), T(t) \mathcal{D}(P) \subset \mathcal{D}(P), T(t) P x=P T(t) x$ for $x \in \mathcal{D}(P)$, and $T(t): \mathcal{N}(P) \rightarrow \mathcal{N}(P)$ has the bounded inverse $T_{Q}(-t)$. Moreover, $T(t) P(w-A)^{-\alpha} x$ and $T_{Q}(-t) Q(w-A)^{-\alpha} x, t \geq 0$, are given by (2.8) and (2.2) with $a=0$ if $x \in \mathcal{D}(A)$, and these operators are uniformly bounded for $t \geq 0$.

We use these formulas in order to derive the desired polynomial decay estimates, starting with the stable case. First observe that we can replace $\alpha$ by $\alpha+\gamma$ in the above reasoning. Let $x \in \mathcal{D}(A)$ and $t \geq 1$. Then we obtain

$$
\begin{aligned}
t T(t) P(w-A)^{-\alpha-\gamma} x= & \frac{1}{2 \pi i} \int_{-i \infty}^{+i \infty}\left(\frac{d}{d \lambda} e^{\lambda t}\right)(w-\lambda)^{-\alpha-\gamma} R(\lambda, A) x d \lambda \\
= & \frac{1}{2 \pi i} \int_{-i \infty}^{+i \infty} e^{\lambda t}(w-\lambda)^{-\alpha-\gamma} R(\lambda, A)^{2} x d \lambda \\
& -\frac{\alpha+\gamma}{2 \pi i} \int_{-i \infty}^{+i \infty} e^{\lambda t}(w-\lambda)^{-\alpha-\gamma-1} R(\lambda, A) x d \lambda \\
= & : J_{1}+J_{2},
\end{aligned}
$$


integrating by parts in the second equality. It is clear that $\left\|J_{2}\right\|$ is less than $c\|x\|$.

In order to estimate $J_{1}$, we will use a duality argument. The dual space $X^{*}$ has the same Fourier type as $X$ by $\left[9\right.$, Prop. 2.3]. Let $X^{\odot}$ be the space of those $x^{*} \in X^{*}$ such that $t \mapsto T(t)^{*} x^{*}$ is continuous in $X^{*}$. The space $X^{\odot}$ is $T(\cdot)^{*}$-invariant and closed in $X^{*}$ (possibly $X^{*} \neq X^{\odot}$ ), and the restriction $T(\cdot)^{\odot}$ of $T(\cdot)^{*}$ to $X^{\odot}$ is generated by the part $A^{\odot}$ of $A^{*}$ in $X^{\odot}$ (see e.g. [8, §II.2.6]). Moreover, the growth bounds of $T(\cdot), T(\cdot)^{*}$, and $T(\cdot)^{\odot}$ coincide by [8, Prop. IV.2.18], and $R\left(\lambda, A^{\odot}\right)$ is the restriction of $R\left(\lambda, A^{*}\right)$ to $X^{\odot}$ for $\operatorname{Re} \lambda>\omega_{0}(A)$. Note that also $X^{\odot}$ has Fourier type $p$. As in Step 1 of Section 2, we thus obtain

$$
\left\|R\left(\widetilde{w}+i \cdot, A^{*}\right) x^{*}\right\|_{L^{p^{\prime}}\left(\mathbb{R}, X^{*}\right)} \leq c\left\|x^{*}\right\|
$$

for $x^{*} \in X^{\odot}$. Finally, due to (II.2.1) of [8] we have

$$
\|y\| \leq \sup \left\{\left|\left\langle y, x^{*}\right\rangle\right|: x^{*} \in X^{\odot},\left\|x^{*}\right\| \leq M\right\}
$$

for $y \in X$, where $\|T(t)\| \leq M e^{w t}$ for $t \geq 0$. We take $x^{*} \in X^{\odot}$ with $\left\|x^{*}\right\| \leq M$. First let $1<p<2$. Proceeding as in (2.5), we can estimate

$$
\begin{aligned}
\left|\left\langle J_{1}, x^{*}\right\rangle\right| \leq & c \int_{\mathbb{R}}|w-i \tau|^{-\alpha-\gamma}\left|\left\langle R(i \tau, A) x, R\left(i \tau, A^{*}\right) x^{*}\right\rangle\right| d \tau \\
\leq & c \int_{\mathbb{R}}(1+|\tau|)^{-\alpha+\gamma}\|R(\widetilde{w}+i \tau, A) x\|\left\|R\left(\widetilde{w}+i \tau, A^{*}\right) x^{*}\right\| d \tau \\
\leq & c\left[\int_{\mathbb{R}}(1+|\tau|)^{(\gamma-\alpha) p /(2-p)} d \tau\right]^{(2-p) / p} \\
& \times\left[\int_{\mathbb{R}}\|R(\widetilde{w}+i \tau, A) x\|^{p^{\prime} / 2}\left\|R\left(\widetilde{w}+i \tau, A^{*}\right) x^{*}\right\|^{p^{\prime} / 2} d \tau\right]^{2 / p^{\prime}} \\
\leq & c\|R(\widetilde{w}+i \tau, A) x\|_{L^{p^{\prime}}(\mathbb{R}, X)}\left\|R\left(\widetilde{w}+i \tau, A^{*}\right) x\right\|_{L^{p^{\prime}}\left(\mathbb{R}, X^{*}\right)} \\
\leq & c\|x\|\left\|x^{*}\right\| \leq c M\|x\|
\end{aligned}
$$

where we have used Hölder's inequality and the relation

$$
\frac{p(\gamma-\alpha)}{2-p}<\frac{p\left(p-p^{\prime}\right)}{(2-p) p p^{\prime}}=-1
$$

As a result, also $\left\|J_{1}\right\|$ is bounded by $c\|x\|$. If $p=2$, the above estimates work even in the case $\alpha=\gamma$. The proof for $p=1$ is similar but simpler. One can treat $T_{Q}(-t) Q$ in the same way. So we obtain the following result.

Theorem 3.1. Let $A$ be the generator of a $C_{0}$-semigroup $T(\cdot)$ on a $B a$ nach space $X$ with Fourier type $p \in[1,2]$. Suppose that $i \mathbb{R} \subset \varrho(A)$ and $\|R(i \tau, A)\| \leq C\left(1+|\tau|^{\gamma}\right)$ for $\tau \in \mathbb{R}$ and constants $C, \gamma>0$. Let $\alpha>$ $1 / p-1 / p^{\prime}+\gamma$. Then there is a closed projection $P$ on $X$ such that $X_{\alpha} \hookrightarrow \mathcal{D}(P)$, 
$T(t) \mathcal{D}(P) \subset \mathcal{D}(P), T(t) P x=P T(t) x$ for $x \in \mathcal{D}(P), T(t): \mathcal{N}(P) \rightarrow \mathcal{N}(P)$ has the bounded inverse $T_{Q}(-t)$, and

$$
\begin{aligned}
\|T(t) P x\| & \leq \frac{N(\alpha)}{t}\left\|(w-A)^{\alpha+\gamma} x\right\|, \\
\left\|T_{Q}(-t) Q x\right\| & \leq \frac{N(\alpha)}{t}\left\|(w-A)^{\alpha+\gamma} x\right\|
\end{aligned}
$$

for $t \geq 1, x \in X_{\alpha+\gamma}$, and a constant $N(\alpha) \geq 0$. If $p=2$ we may take $\alpha=\gamma$ in (3.1). We further have

$$
\begin{aligned}
\|T(t) P x\| & \leq N^{\prime}(\alpha)\left\|(w-A)^{\alpha} x\right\|, \\
\left\|T_{Q}(-t) Q x\right\| & \leq N^{\prime}(\alpha)\left\|(w-A)^{\alpha} x\right\|
\end{aligned}
$$

for $t \geq 0, x \in X_{\alpha}$, and a constant $N^{\prime}(\alpha) \geq 0$.

If $T(\cdot)$ is a bounded semigroup, we obtained a similar result in Theorem 3.5 of [3] with $P=I$ and an arbitrary $\alpha>0$ in (3.1). The difference in the regularity exponent is caused by the unboundedness of $T(t) P$ in Theorem 3.1 .

Under the assumptions of Theorem 3.1, we easily obtain decay estimates on the spaces $X_{\beta}$ for each $\beta>0$. First observe that $T(t) P$ and $T_{Q}(-t) Q$ commute with fractional powers (say, on $X_{n}$ for a sufficiently large $n \in \mathbb{N}$ ). Using (3.1), (3.2), and the moment inequality (see e.g. [8, Thm. II.5.34]), we then deduce for $0<\theta<1$ and $t \geq 1$ that

$$
\begin{aligned}
\left\|T(t) P(w-A)^{-(\alpha+\theta \gamma)}\right\| & \leq c(\alpha) t^{-\theta}, \\
\left\|T_{Q}(-t) Q(w-A)^{-(\alpha+\theta \gamma)}\right\| & \leq c(\alpha) t^{-\theta} .
\end{aligned}
$$

Second, since

$$
\begin{aligned}
T(t) P x & =T(t / n) P \cdots T(t / n) P x, \\
T_{Q}(-t) Q x & =T_{Q}(-t / n) Q \cdots T_{Q}(-t / n) Q x,
\end{aligned}
$$

the estimates (3.1) yield

$$
\begin{aligned}
\|T(t) P x\| & \leq c(\alpha, n) t^{-n}\left\|(w-A)^{(\alpha+\gamma) n} x\right\|, \\
\left\|T_{Q}(-t) Q x\right\| & \leq c(\alpha, n) t^{-n}\left\|(w-A)^{(\alpha+\gamma) n} x\right\|
\end{aligned}
$$

for $n \in \mathbb{N}, t \geq 1$, and $x \in X_{n(\alpha+\theta)}$. Interpolating once more, we arrive at

$$
\begin{aligned}
\left\|T(t) P(w-A)^{-\theta(\alpha+\gamma)}\right\| & \leq c(\alpha, \theta) t^{-\theta}, \\
\left\|T_{Q}(-t) Q(w-A)^{-\theta(\alpha+\gamma)}\right\| & \leq c(\alpha, \theta) t^{-\theta}
\end{aligned}
$$

for $\theta>1, t \geq 1$, and a constant $c(\alpha, \theta) \geq 0$.

Acknowledgements. I would like to thank the referee for pointing out a gap in the original proof of Theorem 3.1. 


\section{References}

[1] F. Alabau, P. Cannarsa and V. Komornik, Indirect internal stabilization of weakly coupled evolution equations, J. Evol. Equ. 2 (2002), 127-150.

[2] H. Amann, Linear and Quasilinear Parabolic Problems, Volume 1: Abstract Linear Theory, Birkhäuser, Basel, 1995.

[3] A. Bátkai, K.-J. Engel, J. Prüss and R. Schnaubelt, Polynomial asymptotic stability of evolution equations, Math. Nachr., to appear.

[4] A. Bátkai and R. Schnaubelt, Asymptotic behaviour of parabolic problems with delays in the highest order derivatives, Semigroup Forum 69 (2004), 369-399.

[5] C. Chicone and Y. Latushkin, Evolution Semigroups in Dynamical Systems and Differential Equations, Amer. Math. Soc., 1999.

[6] R. deLaubenfels, Existence Families, Functional Calculi and Evolution Equations, Lecture Notes in Math. 1570, Springer, 1994.

[7] R. deLaubenfels and Y. Latushkin, Dichotomy and $H^{\infty}$ functional calculi, Electron. J. Differential Equations 1995, no. 13.

[8] K.-J. Engel and R. Nagel, One-Parameter Semigroups for Linear Evolution Equations, Springer, 2000.

[9] M. Girardi and L. Weis, Operator-valued Fourier multiplier theorems on Besov spaces, Math. Nachr. 251 (2003), 34-51.

[10] M. Haase, A general framework for holomorphic functional calculi, Proc. Edinburgh Math. Soc. (2) 48 (2005), 423-444.

[11] M. Hieber, A characterization of the growth bound of a semigroup via Fourier multipliers, in: G. Lumer and L. Weis (eds.), Evolution Equations and their Applications in Physical and Life Sciences (Bad Herrenalb, 1998), Lecture Notes in Pure and Appl. Math. 215, Dekker, 2001, 121-124.

[12] M. A. Kaashoek and S. M. Verduyn Lunel, An integrability condition on the resolvent for hyperbolicity of the semigroup, J. Differential Equations 112 (1994), 374-406.

[13] Y. Latushkin and F. Räbiger, Operator valued Fourier multipliers and stability of strongly continuous semigroups, Integral Equations Operator Theory 51 (2005), 375-394.

[14] Y. Latushkin and R. Shvidkoy, Hyperbolicity of semigroups and Fourier multipliers, in: A. A. Borichev and N. K. Nikolski (eds.), Systems, Approximation, Singular Integral Operators, and Related Topics (Bordeaux, 2000), Oper. Theory Adv. Appl. 129, Birkhäuser, 2001, 341-363.

[15] G. Lebeau and E. Zuazua, Decay rates for the three-dimensional linear system of thermoelasticity, Arch. Rat. Mech. Anal. 148 (1999), 179-231.

[16] A. McIntosh, Operators which have an $H_{\infty}$ functional calculus, in: B. Jefferies, A. McIntosh and W. Ricker (eds.), Miniconference on Operator Theory and Partial Differential Equations (North Ryde, 1986), Proc. Centre Math. Anal. Austral. Nat. Univ. 14, Canberra, 1986, 210-231.

[17] A. McIntosh and A. Yagi, Operators of type $\omega$ without a bounded $H_{\infty}$ functional calculus, in: I. Doust, B. Jefferies, C. Li and A. McIntosh (eds.), Miniconference on Operators in Analysis (Sydney, 1989), Proc. Centre Math. Anal. Austral. Nat. Univ. 24, Canberra, 1990, 159-172.

[18] R. Nagel (ed.), One-Parameter Semigroups of Positive Operators, Lecture Notes in Math. 1184, Springer, 1986.

[19] J. M. A. M. van Neerven, The Asymptotic Behaviour of Semigroups of Linear Operators, Oper. Theory Adv. Appl. 88, Birkhäuser, 1996. 
[20] J. M. A. M. van Neerven, B. Straub and L. Weis, On the asymptotic behaviour of a semigroup of linear operators, Indag. Math. (N.S.) 6 (1995), 453-476.

[21] J. Prüss, Evolutionary Integral Equations and Applications, Birkhäuser, 1993.

[22] S. Schweiker, Asymptotics, regularity and well-posedness of first-and second-order differential equations on the line, Ph.D. thesis, Univ. of Ulm, 2000.

[23] M. Slemrod, Asymptotic behavior of $C_{0}$ semi-groups as determined by the spectrum of the generator, Indiana Univ. Math. J. 25 (1976), 783-792.

[24] L. Weis, Stability theorems for semi-groups via multiplier theorems, in: M. Demuth and B.-W. Schulze (eds.), Differential Equations, Asymptotic Analysis, and Mathematical Physics (Potsdam, 1996), Akademie-Verlag, Berlin, 1997, 407-411.

[25] L. Weis and V. Wrobel, Asymptotic behavior of $C_{0}$-semigroups in Banach spaces, Proc. Amer. Math. Soc. 124 (1996), 3663-3671.

FB Mathematik und Informatik

Martin-Luther-Universität

06099 Halle, Germany

E-mail: schnaubelt@mathematik.uni-halle.de

Received May 11, 2005

Revised version October 27, 2005 